\title{
Labyrinthe
}

19 | 2004 (3)

Le Bel Aujourd'hui

\section{Littérature et musique}

Quelques aspects de l'étude de leurs relations*

\section{Michel Gribenski}

\section{(2) OpenEdition}

Journals

Édition électronique

URL : http://journals.openedition.org/labyrinthe/246

DOI : $10.4000 /$ labyrinthe. 246

ISSN : 1950-6031

Éditeur

Hermann

\section{Édition imprimée}

Date de publication : 15 décembre 2004

Pagination : 111-130

\section{Référence électronique}

Michel Gribenski, « Littérature et musique », Labyrinthe [En ligne], 19 | 2004 (3), mis en ligne le 19 juin 2008, consulté le 19 avril 2019. URL : http://journals.openedition.org/labyrinthe/246 ; DOI : 10.4000/ labyrinthe.246

Propriété intellectuelle 


\title{
LITTÉRATURE ET MUSIQUE Quelques aspects de l'étude de leurs relations*
}

\author{
Michel GRIBENSKI \\ gribenski@hotmail.com
}

Musique et littérature: l'association de ces deux mots suscite immédiatement de nouvelles associations - poésie, chant, rythme, voix, musicalité, mélodie, harmonie, timbre, chanson... Les notions et les genres se rencontrent, se croisent: sous le signe de l'analogie, de la correspondance - ou bien du mariage, voire de la fusion? Si d'aucuns voient, dans certaines œuvres vocales, une conjonction parfaite des deux arts, le compositeur Paul Dukas répond péremptoirement: «Véritablement, vers et musique ne se mêlent pas; ils ne se confondent jamais. [...] On ne met pas les poèmes en musique. On donne un accompagnement aux paroles, et c'est bien autre chose. La première idée, en effet, suppose une fusion; la seconde constate un parallélisme ${ }^{1}$.» Recourant de son côté à l'image de la greffe, Debussy évoque une «musique [qui] commence là où la parole est impuissante à expri$\operatorname{mer}^{2}$ », allant jusqu'à déclarer (non sans provocation) : «Le rapport du vers et de la musique? Je n'y ai pas pensé3. » En deçà même de la relation esthétique entre littérature et musique se pose la question de la relation sémiotique entre deux langages, musique et parole (ou verbe). À Boris de Schlœzer, selon lequel «il y a incompatibilité, opposition

\footnotetext{
* Thèse de littérature comparée sous la direction du professeur Jean-Louis Backès, université Paris IV-Sorbonne, intitulée: «Parole et chant entre vers et prose. Diction et prosodie musicale françaises et allemandes au tournant des XIX et XX ${ }^{\mathrm{e}}$ siècles. Sous le signe de Debussy et de Wagner. »

1. Paul Dukas (1865-1935), revue Musica, mars 1911, réponse à l'enquête de Fernand Divoire: «Sous la musique, que faut-il mettre? [...]» (souligné par Paul Dukas).

2. Entretiens avec son ancien professeur Ernst Guiraud, cités dans Lockspeiser, Edward et Harry Halbreich, Claude Debussy, Paris, Fayard, 1980, p. 703. L'image de la greffe apparaît dans le portrait du librettiste idéal : «Celui qui, disant les choses à demi, me permettra de greffer mon rêve sur le sien. »

3. Claude Debussy, revue Musica, mars 1911, réponse à l'enquête de Fernand Divoire, déjà citée ; reprise dans Claude Debussy, Monsieur Croche et autres écrits, Paris, Gallimard, «L'Imaginaire », 1987 (1 ère éd. 1971), p. 206-207.
} 
profonde entre le langage et la musique ${ }^{4} »$, Nicolas Ruwet répond en linguiste qu' «il n'y a au contraire aucune incompatibilité entre musique et langage, [que] la relation musique-langage est toujours pertinente ${ }^{5} \gg$. Comment, dès lors, penser la relation unissant (selon quelles modalités?) les deux arts, les deux formes d'expression, verbale et musicale?

Il ne saurait bien évidemment être question, dans un texte aussi bref, de traiter de cette relation d'une façon exhaustive, sous tous ses aspects. Notre but est ici d'esquisser un certain nombre de directions possibles dans les recherches musico-littéraires et de déterminer quelle place occupe notre propre recherche sur les prosodies verbale et musicale. Cette thèse, qui s'inscrit dans le champ des études musico-littéraires, vise à mettre au jour les relations entre rythmes verbal et musical à la fin du XIX siècle, en France et en Allemagne, à travers les réalisations ou performances qu'en offrent la diction et la prosodie musicale. Alors que les questions de prosodie musicale ne sont, en général, prises en considération que pour donner lieu à des jugements normatifs, prescriptifs ou évaluatifs, opposant bonne et mauvaise prosodies, il nous a semblé pertinent de nous interroger sur ses présupposés, à partir d'un examen de la diction parlée. En quoi la mise en musique d'un texte constitue-t-elle, en partie, une figuration du dire - et de quel dire? En quoi, plus précisément, la prosodie musicale du tournant des XIX ${ }^{\mathrm{e}}$ et $\mathrm{XX}^{\mathrm{e}}$ siècles reflète-t-elle, accompagne-t-elle et prolonge-t-elle la crise des métriques poétique et musicale, ainsi que celle de la diction? Cette problématique d'ensemble nous conduira ici à interroger les notions de naturel et de prosaïsme, en relation avec le couple vers/prose, qui structure la réflexion littéraire - et plus largement esthétique -, jusqu'à sa mise en question lors de la crise métrique du tournant du siècle.

\section{Esquisse d'une histoire des études musico-littéraires : un domaine au carrefour de plusieurs disciplines}

L'étude des relations entre littérature et musique relève institutionnellement de plusieurs disciplines : littérature comparée (voire française),

\footnotetext{
4. Dans Introduction à J.-S. Bach. Essai d'esthétique musicale, Paris, Gallimard, 1947 (rééd. 1979), p. 262.

5. «Fonctions de la parole dans la musique vocale », dans Langage, musique, poésie, Paris, Le Seuil, «Poétique», 1972, p. 53.
} 
musicologie, esthétique, linguistique, métrique. Comment cet objet d'étude s'est-il progressivement constitué?

Il convient tout d'abord de ne pas confondre les relations entre littérature et musique avec l'étude de ces relations. Les relations ellesmêmes sont très anciennes et peuvent être décrites comme une progressive séparation ${ }^{6}:$ de l'Antiquité grecque et du Moyen $\hat{A} \mathrm{e}^{7}$, où musique et poésie formaient une unité, à l'époque moderne où la poésie cesse d'être nécessairement chantée ${ }^{8}$ et où les relations entre poètes et musiciens se font volontiers conflictuelles ${ }^{9}$, cette séparation n'a cessé d'aller s'accentuant. De ce point de vue, le symbolisme apparait à la fois comme un nouvel âge d'or des relations entre poésie et musique et comme le point culminant d'une rivalité, c'est-à-dire d'une séparation - contestation par certains poètes de l'hégémonie de la musique, appropriation de la poésie par certains musiciens, dans la mélodie française en particulier.

Quant à la réflexion sur les relations entre littérature et musique, si elle est présente dès l'Antiquité (avec Platon et Aristote notamment) et durant tout le Moyen Âge, ce n'est que vers le milieu du XVIII' siècle, en Angleterre, puis en Allemagne et plus tardivement en France, qu'elle s'organise en une véritable critique comparée des deux arts, dégagée de l'esthétique générale qui prévalait jusqu'alors. Assez logiquement, ce travail de réflexion semble se développer particulièrement à partir du moment où est consommée la séparation: plus musique et langue

6. Voir Marie Naudin, Évolution parallèle de la poésie et de la musique en France. Rôle unificateur de la chanson, Paris, Nizet, 1968.

7. Voir Roger Dragonetti, La Musique et les lettres. Études de littérature médiévale, Genève, Droz, 1986.

8. À cet égard, comme l'a montré en particulier Gilbert Gadoffre, les références musicales prodiguées par Ronsard dans son Abrégé de l'Art poétique français, sont vagues et chargées de lieux communs, et relèvent plutôt d'une stratégie de conquête des publics, dans le cadre de la concurrence qui l'oppose à Saint-Gelais, que d'un authentique art poétique original (voir Gadoffre, Gilbert, « Ronsard et la relation poésie-musique», dans Ronsard. Colloque de Neuchâtel, éd. par André Gendre, Neuchâtel/Genève, faculté des lettres/Droz, 1987, p. 75-84). Quant à l'Académie de musique et de poésie, fondée par Antoine de Baïf et Thibault de Courville, elle fait long feu.

9. Que l'on pense à l'interdiction prêtée à Hugo de «déposer de la musique le long de [ses] vers », à la déclaration de Lamartine selon laquelle «la musique et la poésie se nuisent en s'associant (citée dans Musica, mars 1911, op. cit. supra note 1) ou encore à la définition polémique du symbolisme par Valéry comme volonté de certains poètes de «reprendre à la musique leur bien» (Variété I, Gallimard, «Idées », p. 87; cette formule reprend celle de Mallarmé dans «Crise de vers»: «[...] nous en sommes là, précisément, à rechercher [...] un art d'achever la transposition, au Livre, de la symphonie ou uniment de reprendre notre bien» (dans Igitur [...], Gallimard, « Poésie », p. 250). 
se séparent, plus il devient problématique et difficile de comprendre leur association ${ }^{10}$. Marquée par le romantisme allemand qui place la musique au sommet des arts (alors que la théorie classique de l'imitation la mettait au troisième rang, derrière la poésie et la peinture), la réflexion esthétique, au XIX ${ }^{\mathrm{e}}$ siècle, fait de la musique le paradigme idéal des arts.

$\mathrm{Au} \mathrm{XX}^{\mathrm{e}}$ siècle, la réflexion sur les relations entre littérature et musique est notamment marquée par les travaux de l'esthéticien Étienne Souriau ${ }^{11}$, des comparatistes Calvin S. Brown ${ }^{12}$ et Steven Paul Scher ${ }^{13}$. Avec le livre marquant de Brown (1948), les recherches musico-littéraires passent ainsi du domaine de l'esthétique comparée dans celui de la littérature comparée, pour s'y développer durant toute la seconde moitié du siècle ${ }^{14}$. Toujours dans le versant littéraire, il est remarquable que, si la linguistique s'intéresse à la question ${ }^{15}$, les études métriques touchant à la musique concernent, à de rares exceptions près ${ }^{16}$, la chanson, le chant populaire ou la comptine, mais rarement à la musique vocale savante (lied, mélodie, opéra, etc.).

Parallèlement, la musicologie a fait des relations entre texte et musique un objet d'étude à part entière, comme en témoigne, depuis

10. On peut lire avec profit, dans cette perspective, l'essai du musicologue et théoricien allemand Carl Dahlhaus sur L'Idée de la musique absolue. Une esthétique de la musique romantique, trad. fr., Genève, Contrechamps, 1997 (éd. orig. all., 1978).

11. La Correspondance des arts. Éléments d'esthétique comparée, Paris, Flammarion, «Bibliothèque de philosophie scientifique», 1947 (rééd. ibid., «Science de l’homme», 1969), 5e partie: «Musique et littérature», p. 145-218 de la nouvelle édition.

12. Music and Literature. A Comparison of the Arts, Athens (Georgia, EU), The University of Georgia Press, 1948.

13. Steven Paul Scher, éd., Literatur und Musik. Ein Handbuch zur Theorie und Praxis eines kompositorischen Grenzgebietes, Berlin, Erich Schmidt Verlag, 1984.

14. Ainsi, en 1970, la revue Comparative Literature publie un numéro spécial consacré à littérature et musique, sous la direction de Brown; en 1979, c'est aux relations entre la littérature et les autres arts qu'est consacré le neuvième congrès de l'Association internationale de littérature comparée. Citons, parmi les travaux les plus importants publiés en français durant cette période, ceux de JeanLouis Backès, Jean-Pierre Barricelli, Pierre Brunel, Francis Claudon, Léon Guichard, André Wyss. En sous-titrant son ouvrage qui a pour titre Musique et littérature (Puf, 1994): Essai de poétique comparée, Jean-Louis Backès a relancé depuis une dizaine d'années les recherches poïétiques dans le domaine musico-littéraire.

15. Voir en particulier Nicolas Ruwet, «Fonctions de la parole dans la musique vocale», dans Langage, musique, poésie, Paris, Le Seuil, « Poétique», 1972.

16. Voir les travaux menés conjointement par Jacques Roubaud et Pierre Lusson, en particulier de ce dernier, «Les rapports parole-musique: fondements et programmes possibles pour une discipline à créer», dans la revue Analyse musicale, 1987, 4e trimestre, p. 53-56. 
1984, la section intitulée «Text-Music Relation» (rapport(s) textemusique) de l'une des bibliographies musicologiques de référence ${ }^{17}$. Dans la recherche allemande, les monographies musicologiques consacrées au «Wort-Ton Verhältnis» dans la musique vocale de tel ou tel compositeur se sont multipliées ces dernières années, mais sans que soit généralement développée une réflexion véritablement approfondie sur les fondements linguistiques de la collaboration du verbe et de la musique ${ }^{18}$.

À cette pluralité disciplinaire correspond une diversité de méthodologies, sinon d'objets, chacune des disciplines littéraires et musicales s'intéressant respectivement à l'un ou l'autre aspect - avec, bien souvent, des lacunes qui trahissent un manque de réelle compétence dans l'autre domaine. La question prosodique reste, dans la quasi-totalité des études consacrées aux relations musique/texte, entièrement passée sous silence - les métriciens hésitant visiblement à s'aventurer sur le terrain musicologique, tandis que les musicologues semblent peu enclins à entrer dans des considérations techniques étrangères à leur domaine d'origine, ou bien n'évitent pas toujours les erreurs grossières ${ }^{19}$.

\section{Schéma synchronique des relations entre littérature et musique}

On peut globalement distinguer ${ }^{20}$ trois points de rencontre entre littérature et musique, et, partant, trois perspectives dans les études musico-

17. Voir Doctoral Dissertation in Musicology de Cecil Atkins et Alis Dickinson. Le Répertoire international de littérature musicale (RILM) intègre quant à lui, depuis ses origines dans les années 1960, une rubrique consacrée aux relations entre littérature et autres arts.

18. À l'exception, notamment, de la thèse (Paris IV-Sorbonne) du musicologue français d'origine allemande Gottfried R. Marschall, Métrique et musique en allemand. De la poésie à l'opéra, Paris, Klincksieck, 2000.

19. Ainsi, la quasi-totalité des travaux anglo-saxons traitant de la prosodie dans la musique vocale de Debussy reposent sur des présupposés méthodologiques extrêmement discutables concernant le vers français, dont la nature métrique propre est largement méconnue. Voir par exemple Susan Youens, «Music, verse, and "prose poetry" : Debussy's Trois Chansons de Bilitis », Journal of Musicological Research, $\mathrm{n}^{\circ} 7 / 1$, novembre 1986, p. 69-94, et Marie Rolf, «Debussy, Gautier, and "Les Papillons" », dans Debussy and His World, Jane F. Fulcher éd., Princeton UP, 2001, p. 99-115, qui proposent toutes deux des scansions fantaisistes et non justifiées.

20. Nous nous inspirons ici, pour les discuter, des classifications proposées par Calvin S. Brown et par Steven Paul Scher dans leurs ouvrages respectifs, cités ci-dessus. Ces classifications ont été résumées et analysées par Isabelle Piette dans Littérature et musique. Contribution à une orientation théorique (1970-1985), Namur (Belgique), Presses universitaires de Namur, 1987. 
littéraires : collaboration de la musique et de la littérature, présence de la musique dans la littérature, enfin présence de la littérature dans la musique.

La collaboration de la musique et de la littérature se manifeste de façon évidente dans la musique vocale, à travers différents genres, lyriques et dramatiques ${ }^{21}$. Il faut toutefois préciser que, quand le texte littéraire existe précédemment à et indépendamment de la musique - ce qui est le cas le plus fréquent dans la mélodie et le lied, avant d'être le critère définitoire de ce que l'on a appelé le Literaturoper -, on ne saurait proprement parler de collaboration entre poète et musicien (ou alors de collaboration à distance), mais de mise en musique d'un texte, processus complexe d'intégration d'un texte à un autre, en même temps que lecture d'un texte par un compositeur. Cette lecture et, par suite, l'étude des relations entre texte et musique dans la musique vocale, concerne à la fois le plan symbolique, c'est-à-dire le sens du texte (comment le compositeur en rend-il la tonalité générale, les images, etc. ?), et, par ailleurs, tous les aspects du signifiant (accentuation, rythme, etc.), c'est-à-dire la prosodie. Le cas particulier de Wagner, qui écrit lui-même ses poèmes d'opéra (comme le feront, à la fin du $\mathrm{XIX}^{\mathrm{e}}$ siècle, un certain nombre d'autres compositeurs, notamment français $^{22}$ ), est emblématique d'un désir de relation plus étroite, idéalement de fusion entre verbe et musique. L'œuvre vocal de Debussy présente, quant à lui, tous les cas de figure quant au statut du texte : texte autonome préexistant (ainsi de la plupart des mélodies, mais aussi de Pelléas et Mélisande, l'un des tout premiers Literaturoper); texte écrit par le compositeur en vue de sa mise en musique - et, dans ce second cas de figure, (semi-)existence du texte dans la seule partition ou, plus rarement, publication séparée lui donnant une existence littéraire autonome ${ }^{23}$.

21. Précision terminologique importante: on entend ici «lyrique» au sens de poésie lyrique, d'où, musicalement, lied et mélodie; l'opéra, traditionnellement qualifié de genre lyrique, relève à l'inverse, dans cette perspective, du genre dramatique, tout comme la cantate et l'oratorio.

22. Notamment Vincent d'Indy, Albéric Magnard et Ernest Chausson.

23. Ainsi, le texte littéraire des deux premières Proses lyriques, dû à Debussy lui-même, fit l'objet d'une publication dans la revue Entretiens politiques et littéraires, en décembre 1892, avant la composition des mélodies, le texte des deux autres n'apparaissant, en revanche, que dans l'espace de la partition musicale. 
La présence de la musique dans la littérature peut se manifester de façon thématique ${ }^{24}$, par référence à des musiciens réels ou fictifs (Consuelo de George Sand, La Montagne magique de Thomas Mann) ou à des œuvres, elles aussi réelles ou fictives et pouvant alors faire l'objet d'une transposition d'art (Gambara et Massimilla Doni de Balzac); mais elle peut aussi se marquer de façon structurelle, par analogie avec des formes musicales ${ }^{25}$ : c'est ainsi que l'on a pu parler de forme sonate dans Tonio Kröger de Thomas Mann ou dans Steppenwolf de Hermann Hesse; de fugue dans Todesfuge de Paul Celan ou dans Dream Fugue de De Quincey; de thème et variations à propos d'Exercices de style de Queneau ou des Variationen auf eine Hölderlinsche Ode de Josef Weinheber; enfin (et de façon non exhaustive), de forme rondo à propos du chapitre des sirènes, dans l'Ulysse de Joyce. De façon plus générale, la recherche d'une «musicalité » dans la poésie (en vers ou en prose) ou dans le récit poétique, a parfois été interprétée comme la recherche d'analogon musicaux, emblématisée par le poème de Mallarmé, Un coup de dés jamais n'abolira le hasard. Il importe toutefois, dans ce domaine, de toujours prendre garde de marquer la frontière entre analogie et identité sous peine de sombrer dans le confusionnisme.

Enfin, la littérature peut être présente dans la musique à travers la musique à programme, parmi laquelle Calvin S. Brown distingue la musique descriptive et la musique narrative ${ }^{26}$. On peut ici ajouter le paratexte verbal qui fait partie d'une partition musicale (titres, mais aussi indications de caractère, de mouvement, de nuance, etc. ${ }^{27}$ ) et qui constituent bien une présence littéraire dans la musique ${ }^{28}$.

24. Voir par exemple François Sabatier, La Musique dans la prose française. Évocations musicales dans la littérature d'idée, la nouvelle, le conte ou le roman français des Lumières à Marcel Proust, Paris, Fayard, 2004.

25. Voir en particulier Steven Paul Scher, éd., op. cit., et Music and Text : Critical Inquiries, Cambridge University Press, 1992.

26. Sur la question de la narrativité dans la musique, voir Carolyn Abbate, Unsung Voices, Princeton, Princeton University Press, 1991 (inédit en français).

27. Voir en particulier les recherches de Françoise Escal, notamment dans Contrepoints. Musique et littérature, Paris, Klincksieck, 1990.

28. Dans un article plus récent, C. S. Brown propose une quadripartition quelque peu différente, en distinguant «combinaison», « remplacement», «influence» et «parallèle ou analogie». Moins schématique et séduisant à première vue, ce modèle est peut-être finalement moins efficace que le premier pour penser les relations entre littérature et musique. En effet, si combinaison et remplacement corres- 


\section{Place des recherches prosodiques}

Les recherches prosodiques, peu nombreuses encore et que je propose de nommer «prosodologie», se situent donc au cœur de la collaboration effective de la littérature et de la musique dans la musique vocale, de la façon la plus concrète, tangible et technique qui soit. La question est alors celle de la relation entre diction parlée et prosodie musicale. Dans quelle mesure la mise en musique d'un texte par un compositeur constitue-t-elle une figuration du dire?

On peut définir la prosodie musicale comme le traitement musical du signifiant verbal, sous l'angle de l'accentuation, de la syllabation (notamment en ce qui concerne l' ' $\mathrm{e}$ » caduc et les diérèses), du débit et du profil intonatif et expressif.

De nombreux problèmes se posent, et en premier lieu des problèmes liés à l'interprétation. Comment dire un texte? Dit-on de la même façon un texte en prose et un texte en vers? Doit-on réaliser tous les «e » sourds, dans le corps du vers, en fin de vers (distinction des rimes féminines et masculines)? Doit-on marquer un accent et/ou une pause à la césure, et en fin de vers - quelle lecture adopter dans les cas d'enjambements ? Comment, parallèlement, interpréter - c'est-à-dire réaliser ${ }^{29}$ une partition musicale sur le plan de l'accentuation, du phrasé, etc.? Comment, dans les deux cas, déterminer l'accent ou plutôt les différents types d'accents?

On est également confronté à un problème terminologique, plusieurs termes identiques renvoyant, en littérature et en musique, à des notions et réalités parfois différentes : mesure, rythme, mètre - sans parler de termes musicaux métaphoriquement appliqués à la littérature et singulièrement à la poésie (mélodie, harmonie, contrepoint, etc.).

Le point de départ des recherches prosodiques doit logiquement concerner la diction parlée. Il s'agit de mettre au jour, à partir de trai-

pondent respectivement à musique vocale (collaboration) et à musique à programme (présence de la littérature dans la musique), les notions d'influence et de parallèle ou analogie tendent à s'interpénétrer de façon problématique, intéressante, mais non sans entraîner quelque confusion.

29. Littérature - poésie et théâtre principalement - et musique savante ont en commun une double nature écrite (en puissance) et réalisée sous forme de performance orale (en acte). En dépit du dogme inspiré de Valéry selon lequel la poésie n'existerait que réalisée, on peut opposer la poésie comme pouvant être réalisée à la musique comme devant l'être. La place manque ici pour justifier ce point de vue, méthodologiquement important. Voir aussi la critique de ce «mythe de l'universelle oralité poétique» par Benoît de Cornulier, dans Art poëtique. Notions et problèmes de métrique, Lyon, Presses universitaires de Lyon, «IUFM», 1995, p. 14-17. 
tés de diction et de versification, d'ouvrages scientifiques, d'articles (spécialisés ou de grande diffusion), mais aussi, le cas échéant, d'enregistrements vocaux, l'état, ou plutôt les états de la diction parlée - poétique, dramatique, soutenue ou courante, voire familière - à une époque donnée, afin d'étudier ensuite dans quelle mesure la prosodie musicale de la même époque est en phase ou au contraire en décalage avec elle.

Les partitions de musique vocale constituent à la fois un témoin de la diction pratiquée à une époque donnée, et une lecture singulière s'inscrivant de façon harmonieuse ou conflictuelle avec la tendance générale de cette époque. C'est généralement sous le premier aspect qu'elles ont été convoquées par les commentateurs. Ainsi, Romain Rolland, se demandant en quoi l'étude de la musique vocale prosodie musicale pourrait intéresser la littérature, répond à cette question dans son étude sur la déclamation de Lully: «L'histoire littéraire n'a pas encore tiré de l'histoire musicale tous les secours qu'elle pourrait y trouver. Bien des problèmes littéraires seraient plus faciles à résoudre, s'ils s'éclairaient de la musique. [...] Les musiciens ont, plus ou moins sciemment, transposé en musique la façon de déclamer de leur temps; et, à travers leurs chants, nous percevons encore la voix des grands acteurs qui étaient leurs modèles, ou qui faisaient loi, autour d'eux ${ }^{30}$.» C'est à partir du même type d'hypothèses que Georges Lote, l'un des pionniers de la phonétique expérimentale, utilise nombre de documents musicaux (notamment les opéras de Lully, Rameau ou Grétry) dans son Histoire du vers français. Nous considérerons quant à nous les partitions musicales, non comme de simples témoins, mais comme des lectures singulières de textes, engagées dans une relation problématique à une diction parlée elle-même problématique.

Les recherches concernant la prosodie musicale, très peu nombreuses, sont le plus souvent marquées par un flou méthodologique et une normativité qui en limitent la portée critique. Les quelques traités ${ }^{31}$ consacrés à cette question visent en effet le plus souvent, dans une optique prescriptive, à relever des fautes de prosodie, sans se poser la question des possibles de la diction parlée, ni celle des choix du compo-

30. Romain Rolland, « Notes sur Lully», Mercure musical et SIM [Société internationale de musique], 15 janvier 1907, p. 4-5.

31. Voir, en particulier, Henry Woollett (1903), Paul Rougnon (s. d.) et Émile Stévenard (1924). 
siteur. Ces recherches souffrent de plus d'une lacune quasi complète à l'endroit de la versification, alors même que, jusqu'à la toute fin du XIX siècle, tous les livrets d'opéra, tous les poèmes servant de support aux lieder et aux mélodies, étaient écrits dans la langue contrainte des vers, qui pose des problèmes particuliers touchant leur mise en musique.

\section{Quelques aperçus d'une recherche en cours. Choix d'un corpus, d'une période et d'une problématique : l'exemple de Debussy et de Wagner}

Il importait, pour la pertinence de l'étude prosodique, de délimiter un corpus significatif. Le tournant des $\mathrm{XIX}^{\mathrm{e}}$ et $\mathrm{XX}^{\mathrm{e}}$ siècles est intéressant car il correspond à une période de crise métrique, tant en poésie qu'en musique.

\section{Crise métrique}

Dans le domaine de la versification française, le XIX ${ }^{e}$ siècle est en effet marqué par une évolution (parfois idéologiquement caractérisée comme une «libération») qui voit la remise en cause de la coïncidence entre syntaxe et grandes articulations métriques, fin de vers et surtout césure. L'apparition du vers dit «libre», au cours des années 1880, constitue l'un des symptômes de la «crise de vers » diagnostiquée par Mallarmé, et culminant en 1913 avec la publication d'Alcools d'Apollinaire: ainsi, le premier vers du recueil, à l'attaque de Zone: «À la fin je suis las de ce monde ancien »- avec sa problématique diérèse qui fait signe précisément vers ce «monde ancien» de la versification traditionnelle est emblématique de l'élasticité métrique qui va caractériser la versification du $\mathrm{XX}^{\mathrm{e}}$ siècle. Or, la diction, au tournant du siècle, va également dans le sens de l'élasticité du nombre syllabique, comme en témoignent les travaux de phonétique expérimentale ${ }^{32}$, un certain nombre de trai-

32. Voir Georges Lote, L'Alexandrin d'après la phonétique expérimentale, 1913-1914; Histoire du vers français, publié à partir de 1949. Lote montre, à partir de tracés mesurant des déclamations de divers contemporains, que le nombre de syllabes de l'alexandrin, théoriquement de douze, varie en réalité de neuf à quinze. 
tés et études spécialisés, dus notamment à des philologues allemands ${ }^{33}$, ainsi que des enregistrements sonores ${ }^{34}$. Il reste que tout un débat, émaillé de polémiques concernant singulièrement $l^{\text {'«e }}$ » dit «muet ${ }^{35}$ » qui fait alors décidément grand bruit, entoure la question de la diction au tournant du siècle.

Parallèlement (coïncidence significative), on assiste, dans le domaine de la musique, à la remise en cause de la «tyrannie de la mesure ${ }^{36} »$, c'est-à-dire de la périodicité métrique avec retour régulier du temps fort - remise en cause corrélative de celle du système mélodico-harmonique, qui conduira dans certains cas à l'atonalité. Alors que la construction de la phrase musicale était, depuis le milieu du XVIII ${ }^{\mathrm{e}}$ siècle au moins, régie par les lois de la symétrie, techniquement dénommée «carrure», le romantisme a introduit des formes d'irrégularité et d'asymétrie, qui vont s'approfondissant tout au long du XIX siècle. Chopin, Liszt et Wagner sont les principaux artisans de cet assouplissement métrique, auquel contribueront aussi fortement, dans le domaine français, Fauré, Debussy ou Ravel, au profit d'une rythmique beaucoup plus fluide.

\section{Vers et prose}

Face à cette crise métrique se pose la question du couple vers/prose. Sur le plan littéraire, la distinction entre vers et prose, paradoxalement relégitimée par le poème en prose (qui dissocie poésie et vers, mais sans identifier vers et prose), se voit contestée par le vers libre qui, au sein même du vers, remet radicalement en cause, comme critères défini-

33. Voir en particulier Adolphe Mende, Études sur la prononciation de l' «e» mиet à Paris, 1880; Sonnenburg R., Wie sind die französische Verse zu lesen?, 1885.

34. Voir les enregistrements de Sarah Bernhardt, notamment dans Phèdre: non que ces derniers soient prosaïques au sens large du terme, mais la fréquence des apocopes et des diérèses y faussent très souvent la mesure du vers.

35. Voir, en particulier, la polémique qui oppose, en 1894, Jean Psichari et Francisque Sarcey dans Le Temps; ainsi que, en 1904, celle qui oppose M. D. Calvocoressi (traducteur, notamment, de Moussorgski), Jacques d'Offoël (traducteur de Wagner) et Camille Saint-Saëns concernant «Le vers, la prose et l' "e" muet".

36. Cette expression apparaît à plusieurs reprises dans des traités de composition du début du $\mathrm{XX}^{\mathrm{e}}$ siècle, tel celui de Vincent d'Indy (t. 1, publié en 1912, d'après des notes de cours de 1897-1898, p. 27). Voir aussi Philippe Biton, Le Rythme musical [...], 1948, et René Dumesnil, Le Rythme musical [...], 1949 (rééd. 1979). 
toires, la pertinence des notions de mètre, de nombre syllabique et de rime. Mallarmé pousse la logique jusqu'à nier, l'un des tout premiers, la distinction entre vers et prose, au profit du vers : «Le vers est partout dans la langue où il y a rythme, partout, excepté dans les affiches et la quatrième page des journaux. Dans le genre appelé prose, il y a des vers, quelquefois admirables, de tous rythmes. Mais, en vérité, il n'y a pas de prose: il y a l'alphabet, et puis des vers plus ou moins serrés, plus ou moins diffus. Toutes les fois qu'il y a effort au style, il y a versification $^{37}$.» Dans le domaine de la diction, les traités posent la question de savoir si, au nom du naturel, il faut dire les vers comme la prose. Les avis sont partagés, concernant notamment la réalisation de l' «e » caduc et de l'enjambement (il est curieusement peu question de la diérèse), mais, comme on l'a vu, un certain nombre de documents attestent la réalité d'une diction prosaïsée du vers au début du $\mathrm{XX}^{\mathrm{e}}$ siècle.

En musique, enfin, la question de la prose intervient de diverses façons, littérale et métaphorique. En quoi la mise en musique d'un texte versifié tend-elle à faire de ce dernier de la prose? Les compositeurs du tournant des $\mathrm{XIX}^{\mathrm{e}}$ et $\mathrm{XX}^{\mathrm{e}}$ siècles font-ils une lecture prosaïsée des vers? Réciproquement, en quoi un texte en prose informe-t-il une «musique en prose» - et que peut-on entendre par cette notion ${ }^{38}$ ? L'analogie - mieux, l'isomorphie - entre la symétrie de la phrase musicale et celle du vers métrique est soulignée par certains auteurs ${ }^{39}$, de même que l'association corrélative entre symétrie et vers d'une part, asymétrie et prose de l'autre ${ }^{40}$. À la distinction vers/prose correspond dans l'opéra l'opposition air/récitatif: bien que les récitatifs soient traditionnellement composés sur des textes versifiés, la musique correspond à celle d'une

37. Réponse à l'enquête de Jules Huret sur le vers libre.

38. Sur la notion de prose musicale, voir Hermann Danuser, Musikalische Prosa, Regensburg, G. Bosse, 1975

39. Voir Fétis, «Sur la coupe des vers lyriques et sur la disposition des paroles des divers morceaux de musique dramatique». L'épithète «lyrique» renvoie ici aux vers faits pour la musique; notre épithète «métrique», ici synonyme de régulier ou réglé, vise à distinguer le vers métrique du vers libre, non métrique.

40. Voir par exemple Nicolas Étienne Framery, Discours sur cette question : Analyser les rapports qui existent entre la musique et la déclamation. - Déterminer les moyens d' appliquer la déclamation à la musique, sans nuire à la mélodie (prix de musique et déclamation proposé par l'Institut de France et remporté en 1802). On consultera également avec profit la synthèse de Herbert Schneider pour la seconde moitié du XVIII ${ }^{\mathrm{e}}$ siècle, "Gluck als "prosateur en musique" ", dans Festschrift Klaus Hortschansky zum 60. Geburtstag, Tutzing, H. Schneider, 1995, p. 193-209. 
«prose musicale ${ }^{41}$ 》 - on s'en souviendra à propos du récitatif mélodique de Pelléas, l'un des tout premiers opéras à être composé sur un texte en prose. Dans l'opéra-comique des XVIII et ${ }^{\mathrm{e}}$ XIX ${ }^{\mathrm{e}}$ siècles, les dialogues parlés - en prose - constituent un pendant prosaïque aux airs lyriques; de même, il semble tout à fait significatif que les premiers mélodrames, tant en France (Pygmalion, de Rousseau, ) qu'en Allemagne (ceux de Jiri Benda, notamment), associent à la musique des textes en prose. Dans l'opéra naturaliste, dont le sujet est volontairement non noble, il est remarquable que ce prosaïsme thématique s'accompagne de l'utilisation de la prose: si tous les opéras naturalistes ne sont pas écrits en prose, il reste que c'est le cas de la grande majorité d'entre eux, notamment les œuvres les plus emblématiques, comme Messidor d'Alfred Bruneau (1897) ou Louise de Gustave Charpentier (1900). Cette prose est cependant elle-même diversifiée, avec une distinction entre passages lyriques soutenus (par exemple, l'air initial de Julien dans Louise) et passages populaires (acte II du même opéra). De plus, l'utilisation de la prose y contraste avec des traits paradoxalement non prosaïques de la prosodie des compositeurs naturalistes (réalisation des «e » caducs après voyelle, diérèses), en particulier chez Bruneau, et de façon plus nuancée chez Charpentier, qui utilise la petite note barrée surnuméraire pour noter des «semi-diérèses » et des «semi-apocopes».

Les controverses auxquelles donne lieu l'apparition du livret en prose $^{42}$ sont en réalité davantage centrées sur la question du prosaïsme que sur celle de l'incidence prosodique et/ou musicale de l'utilisation de la prose sur la mise en musique. Quel lien, dès lors, établir entre prose, naturel et prosaïsme?

41. N[icolas] E[tienne] Framery, op. cit., p. 16 : «Dans les productions musicales, comme dans les productions littéraires, on doit reconnaître de la poésie et de la prose; et la prose doit de même être divisée en deux espèces : $1^{\circ}$ la prose poétique $[\ldots] ; 2^{\circ}$ la prose commune [...].»

42. En particulier la polémique qui oppose Zola, auteur du livret de Messidor, à Louis de Fourcaud dans Le Gaulois, 1897. Sur la question du livret en prose, voir Macdonald Hugh, «The Prose Libretto », Cambridge Opera Journal, 1989, n 1/2, p. 155-166, ainsi que Leroy Christian, «Le chant de la prose : remarques sur le statut de la prose dans les livrets d'opéras français entre 1875 et 1914 », dans Le Livret d'opéra au temps de Massenet, Publications de l'université de Saint-Étienne, actes du colloque de novembre 2001, 2003, p. 115-137. Loin de nous contenter de reprendre les aperçus déjà riches de ces auteurs, nous avons au contraire cherché à préciser et à étendre le corpus d'œuvres - opéras, mélodies, cantates - composées sur des paroles en prose, mais aussi et surtout à approfondir l'analyse et la réflexion concernant les relations entre prose littéraire et prose musicale. 


\section{Naturel et prosaïsme}

Depuis les années 1660 environ et la tentative de réforme prônée par Molière et par Racine, la diction théâtrale est comme hantée par la recherche du naturel et par cette question: faut-il prononcer les vers comme la prose? Elle se poursuit tout au long des XVIII et XIX siècles et acquiert une acuité particulière à la fin du XIX ${ }^{e}$ siècle, à la faveur à la fois du naturalisme et du vers libre. Dans la musique vocale française, une tradition prosodique impose la réalisation des «e» caducs, même après voyelle. Or, une recherche du naturel se fait jour, avec Debussy et Ravel, notamment, qui se manifeste en particulier par une tendance à l'apocope: ainsi, dans une note d'avril 1902, intitulée «Pourquoi j'ai écrit Pelléas », Debussy déclare: «[...] les personnages de ce drame tâchent de chanter comme des personnes naturelles et non pas dans une langue arbitraire faite de traditions surannées »; tandis que Ravel compose en 1906 les Histoires naturelles, titre dans lequel l'adjectif est autant de qualité que, si l'on peut dire, de nature. Cependant, la recherche du naturel s'accompagne d'un rejet du prosaïque. Ainsi, Debussy, à propos des mêmes Histoires naturelles, raille «l'américanisme voulu» de son collègue - terme ambigu dans lequel il semble qu'on doive lire une dénonciation de la vulgarité de cette prosodie apocopée. Ce même rejet du prosaïsme est sensible dans le jugement de Romain Rolland concernant la prosodie des compositeurs naturalistes.

Si l'on qualifie de prosaïque, non seulement ce «qui a rapport ou appartient à la prose», mais par suite (au sens figuré), ce qui «manque d'élégance, de distinction, de noblesse - [est] commun, vulgaire ${ }^{43} »$, on peut construire un couple naturel/prosaïsme, dans lequel «naturel» renvoie, de façon méliorative, à la simplicité d'une diction qui ne s'éloigne pas de la parole naturelle (par opposition à l'artifice de la parole esthétisée), tandis que les connotations péjoratives attachées à «prosaïsme» le tirent vers le registre du manque (manque de noblesse, d'élégance, de distinction), selon une idéologie valorisant le naturel et dévalorisant l'ignoble (au sens étymologique de ce qui n'est pas noble, c'est-à-dire ici populaire). Il reste que la notion de naturel s'avère elle-

43. Définition du dictionnaire Le Robert. Littré propose une définition assez proche : $1^{\circ}$ qui tient trop de la prose; $2^{\circ}$ Fig. et néologisme. Vulgaire, sans éclat, en parlant des personnes et des choses. 
même fort problématique, dans la mesure où elle renvoie à des niveaux de langue et à des représentations esthétiques très divers.

Qu'en est-il donc de la question du naturel et du prosaïsme dans Pelléas ou dans les Histoires naturelles de Ravel? Si le caractère symboliste de Pelléas a souvent été souligné, d'autres aspects ne doivent cependant pas être négligés : grande simplicité du lexique et de la syntaxe, traits prosodiques allant dans le sens de la diction familière (apocopes, synérèses), à côté de traits plus traditionnels (réalisation d' «e » caducs après voyelle, diérèses). Les Histoires naturelles représentent, quant à elles, une expérience prosodique limite: si l'apocope n'y est pas systématique, elle y est cependant fréquente et constitue un indice de prosaïsme voulu ${ }^{44}$.

\section{Naturel et perfection de la prosodie debussyste en question}

Le choix de Debussy comme point focal de l'étude sur les relations entre diction parlée et prosodie musicale s'imposait presque, dans la mesure où le compositeur est considéré quasi unanimement comme un modèle absolu et indépassable en matière de prosodie française, tant par sa supposée perfection que par son prétendu naturel. Or, l'étude prosodique de son œuvre vocal fait apparaître le caractère problématique de la notion de naturel prosodique, y compris dans Pelléas et Mélisande: en dépit des revendications de naturel par le compositeur, innovation et tradition prosodiques y sont cependant en perpétuelle tension. Ainsi, Debussy est l'un des tout premiers compositeurs à pratiquer largement l'apocope de l' «e » caduc après voyelle et la synérèse. Par ailleurs, l'étude prosodique des mélodies notamment met au jour, dans le cas de mise en musique de vers métriques, la présence de nombreux écarts par rapport au code de la versification - diérèses non réalisées, «e » apocopés - modifiant dans certains cas le nombre syllabique : faut-il y voir simplement des fautes de prosodie, ou au contraire des traces, dans la prosodie musicale, d'une mutation de la versification et de la diction parlée, liée à la «crise de vers » et allant dans le

44. Le scandale que provoqua la première audition de Histoires naturelles, le 12 juin 1907 salle Érard, était essentiellement dû au choix des textes et à leur traitement prosodique. 
sens d'une élasticité du nombre syllabique? La seconde thèse apparaît non seulement comme la plus fructueuse, mais comme la plus juste: elle fait de Debussy l'artisan d'une « révolution subtile ${ }^{45}$ », permettant de passer de la prosodie traditionnelle à une nouvelle prosodie.

La comparaison avec d'autres compositeurs contemporains de Debussy montre que, à côté de ses innovations comme de la radicalité d'un Ravel, une tradition prosodique, dans le traitement de l'«e » caduc et de la diérèse précisément, continue d'exister, y compris, comme on l'a vu, dans l'opéra naturaliste.

\section{Confrontations wagnériennes}

Il importait, dans une perspective comparatiste, de confronter le corpus français avec un corpus étranger. Parmi différents corpus européens (italien, russe, tchèque, anglais notamment), le corpus allemand s'est imposé en raison de l'influence dominante exercée, en France, par la figure de Wagner à la fin du XIX⿸ siècle, tant sur le plan musical que sur celui des relations entre verbe et musique, et ce du double point de vue théorique et pratique ${ }^{46}$. Le corpus wagnérien montre une évolution conjointe de la versification, de la syntaxe mélodique et de la prosodie vocale: l'évolution de facture du vers wagnérien (du pentamètre iambique traditionnel rimé au Stabreim bref, sans incursion toutefois dans le domaine de la prose littéraire) s'accompagne en effet d'une évolution de la construction musicale, non seulement par l'emploi des leitmotivs, mais aussi par le développement, dans la mélodie infinie, d'une «prose musicale». La conjonction entre versification et construction mélodique métriquement régulières que présentent les opéras de jeunesse se trouve donc inversée dans les opéras de la maturité, qui associent au contraire versification irrégulière (Stabreim au nombre variable de syllabes accentuées) et mélodie infinie.

De plus, la comparaison avec un corpus allemand permet de réfléchir sur la différence, non seulement des systèmes linguistiques (touchant l'accent notamment), mais également des systèmes de versi-

45. Pour reprendre le titre du livre d'André Boucourechliev, Debussy. La Révolution subtile, Fayard, 1996.

46. La réflexion de Wagner sur le langage, en particulier dans Opéra et drame (1851; trad. fr. 1928, rééd. 1982), fait écho de façon frappante à celle de Rousseau, sans que celui-ci soit cité. 
fication français et allemand; plus précisément, l'invention, dès l'époque du Sturm und Drang ${ }^{47}$, du vers libre allemand, au nombre d'accents variable et parfois non rimé, invite à une comparaison musico-littéraire par-delà les siècles. La spécificité française s'en trouve nuancée, puisque l'élasticité du nombre syllabique, caractéristique de la crise de vers française, se retrouve structurellement en allemand. Mais il reste que la fin du XIX ${ }^{\mathrm{e}}$ siècle offre la particularité d'une conjonction avec une crise métrique affectant également la musique, ce qui n'était pas le cas à l'époque du Sturm und Drang. La comparaison a donc ici cette vertu d'éviter d'établir un lien mécanique et figé entre irrégularité du nombre syllabique et irrégularité dans la métrique musicale, mais d'inviter plutôt à penser ce lien dans un contexte général de crise métrique.

\section{Traductions en question}

Le problème de la traduction des «paroles» d'opéras ou de lieder conduit enfin à prolonger et à approfondir la réflexion sur le traitement comparé du français et de l'allemand dans la musique. Pratique non seulement dominante, mais quasi exclusive en France jusqu'à la Seconde Guerre mondiale, la traduction musicale pose des problèmes de méthode liés à la prosodie. Plusieurs options s'offrent au traducteur : soit conserver la ligne mélodique intacte, en rendant une syllabe par une syllabe; soit la modifier selon les besoins de la traduction, en fonction du nombre de syllabes ou de l'accentuation. Le cas des opéras de Wagner offre une palette variée de solutions, illustrant dans le domaine de la traduction ce phénomène beaucoup plus large de crise métrique et prosodique. Alors qu'un Victor Wilder, à la suite de Charles Nuitter ${ }^{48}$, n'hésite pas à modifier la ligne mélodique originale pour obtenir en français des vers pairs rimés, dans la pure tradition du livret d'opéra, un Alfred Ernst maintient au contraire intacte cette ligne mélodique (à quelques très rares exceptions près), obtenant donc, comme Wagner, un nombre irrégulier de syllabes dans le cas de Stabreim et n'ajoutant pas de rimes finales - bref, en traduisant par des vers libres. Les traduc-

47. Il s'agit notamment des poèmes du jeune Goethe, tels que Ganymed ou Prometheus.

48. Nuitter (anagramme de Charles Truinet) est l'auteur des premières traductions chantées des opéras de Wagner, en particulier de celle de Tannhäuser et de Lohengrin. 
tions d'Ernst présentent en outre la particularité - corrélative de la première - de mettre en question une tradition musicale très établie, en pratiquant l'apocope d'un grand nombre d' «e» caducs, après voyelle ou après consonne, manifestant donc, dans le domaine de la traduction musicale, une évolution parallèle à celle de la prosodie poétique et de la diction. Ce choix est entièrement assumé et revendiqué par le traducteur, au nom du naturel : «Lorsqu'elles terminent un membre de phrase, et lorsqu'elles sont suivies d'un signe de ponctuation, d'un signe musical de silence ou d'une respiration nécessaire au chanteur, les syllabes muettes sont traitées comme dans le langage courant et la déclamation dramatique ordinaire $[\ldots]^{49}$.»

Le rejet du prosaïque a la vie dure. C'est ainsi que l'on en trouve plus que des traces dans l'article que Jean-Louis Jam et Gérard Loubinoux consacrent à l'étude de diverses traductions de $L a$ Walkyrie $^{50}$. À propos de la traduction d'Ernst et des apocopes pratiquées par ce dernier, les auteurs portent soudain un jugement de valeur, en commettant au passage plusieurs confusions terminologiques: "Mais hélas! c'est la métrique [la prosodie] française qui en fait les frais car l'interprète est invité à élider [apocoper] le "e" muet [...] et à prononcer « un' source $[\mathrm{sic}]$ » entorse importante, peut-être avant-gardiste, aux règles de la déclamation poétique française. L'effet peut être catastrophique, devant un public pour qui ce type d'élision est la marque indiscutable du vulgaire.» Après avoir cité, comme repoussoir prosodique, un extrait de La Vie parisienne d'Offenbach (tout comme l'avait fait Saint-Saëns dans un article consacré à la prosodie ${ }^{51}$ ), les auteurs de conclure: «C'est là un des choix les plus discutables d'Ernst. » Le flou conceptuel s'accompagne ici de préjugés sociaux et esthétiques, montrant que l'un des enjeux de la prosodie est bien, comme l'a montré Henri Meschonnic à propos de rythme ${ }^{52}$, idéologique et politique.

Par ailleurs, l'étude des versions françaises de la Salomé de Richard Strauss, nourrie par la correspondance entre le compositeur et Romain

\footnotetext{
49. Alfred Ernst, Avertissement à sa «traduction nouvelle en prose rythmée exactement adaptée à la musique » de La Walkyrie, Paris, Schott, ca 1896.

50. «D'une Walkyrie à l'autre... Querelles de traductions », dans Annegret Fauser et Manuela Schwartz éd., Von Wagner zum Wagnérisme, Leipzig, 1999, p. 401-430.

51. «La poésie et la musique», dans Harmonie et mélodie, Paris, Calmann-Levy, 4éd. 1890.

52. Critique du rythme, Paris, Verdier, 1982.
} 
Rolland ${ }^{53}$ sur les problèmes prosodiques, permet d'affiner la comparaison des deux langues et des deux prosodies verbale et musicale, tout en mettant à nouveau au jour les enjeux idéologiques véhiculés par la prosodie.

La confrontation de deux langues, de deux corpus littéraires et musicaux permet donc de nourrir une réflexion sur la question de la prose et des vers, en relation avec des notions aussi problématiques que naturel et prosaïsme. À partir d'une étude technique des relations entre diction parlée et prosodie musicale se trouvent ainsi posées des questions esthétiques centrales, à une époque où, en France, la «crise de vers » est l'expression particulière d'une remise en question plus générale, européenne, de l'ordre métrique, tant poétique que musical.

Cette recherche, qui s'inscrit dans le vaste champ des études musicolittéraires, se concentre donc sur un objet extrêmement technique, permettant de rester au plus près des deux domaines littéraire et musical. Dans une perspective résolument interdisciplinaire, sont convoquées à la fois la linguistique, la métrique, et la musicologie sous son double aspect analytique et historique. Mais plus encore qu'interdisciplinaire, ce travail se veut comparatiste. Comparer, c'est non seulement faire apparaître des similitudes et des différences, mais, de façon beaucoup plus dynamique, mettre en relation, c'est-à-dire en tension, pour faire surgir des spécificités. Or, il s'agit bien, en effet, de faire jouer, l'une par rapport à l'autre, deux formes d'expression (musique et littérature, à la fois arts et langages), mais aussi deux langues (français et allemand), tant par le choix d'un double corpus que par l'étude des processus de traduction des paroles d'une œuvre musicale - il s'agit donc bien, au sein même d'un corpus musico-linguistique donné, de confronter plusieurs lectures musicales d'un même texte, avec le souci de ne pas se contenter de jugements normatifs, mais de mettre au jour la pluralité des choix de lecture. 


\section{BIBLIOGRAPHIE SÉLECTIVE}

BACKES Jean-Louis, Musique et littérature. Essai de poétique comparée, Paris, Puf, «Perspectives littéraires », 1994

Brown Calvin S., Music and Literature. A Comparison of the Arts, Athens (Georgia, EU), the University of Georgia Press, 1948

PIETTE Isabelle, Littérature et musique. Contribution à une orientation théorique (1970-1985), Namur (Belgique), Presses universitaires de Namur, 1987

Scher Steven Paul, éd., Literatur und Musik. Ein Handbuch zur Theorie und Praxis eines kompositorischen Grenzgebietes, Berlin, Erich Schmidt Verlag, 1984

Wyss André, Éloge du phrasé, Paris, Puf, «Écriture», 1999. 